\title{
32.
}

\section{ON SOME ANALYTICAL FORMULA, AND THEIR APPLICATION TO THE THEORY OF SPHERICAL COORDINATES.}

[From the Cambridge and Dublin Mathematical Journal, vol. I. (1846), pp. 22-33.]

\section{SECTION 1.}

THE formulæ in question are only very particular cases of some relating to the theory of the transformation of functions of the second order, which will be given in a following paper. But the case of three variables, here as elsewhere, admits of a symmetrical notation so much simpler than in any other case (on the principle that with three quantities $a, b, c$, functions of $b, c$; of $c$, $a$; and of $a, b$, may symmetrically be denoted by $A, B, C$, which is not possible with a greater number of variables) that it will be convenient to employ here a notation entirely different from that made use of in the general case, and by means of which the results will be exhibited in a more compact form. There is no difficulty in verifying by actual multiplication, any of the equations here obtained.

It will be expedient to employ the abbreviation of making a single letter stand for a system of quantities. Thus for instance, if $\gamma=\theta, \phi, \psi$, this merely means that $\Phi(૪)$ is to stand for $\Phi(\theta, \phi, \psi), k 8$ for $k \theta, k \phi, k \psi$, \&c.

Suppose then

$$
\begin{aligned}
& \omega=\xi, \eta, \zeta \\
& \omega^{\prime}=\xi^{\prime}, \eta^{\prime}, \zeta^{\prime} \\
& \vdots \\
& Q=A, B, C, F, G, H \\
& W\left(\omega, \omega^{\prime}, Q\right)=A \xi \xi^{\prime}+B \eta \eta^{\prime}+C \zeta \zeta^{\prime}+F\left(\eta \zeta^{\prime}+\eta^{\prime} \zeta\right)+G\left(\zeta \xi^{\prime}+\zeta^{\prime} \xi\right)+H\left(\xi \eta^{\prime}+\xi^{\prime} \eta\right)
\end{aligned}
$$

the function $W$ satisfies a remarkable equation, as follows: 
write

$$
\begin{aligned}
& \mathfrak{A}=B C-F^{2}, \\
& \mathbf{a} \mathbf{B}=C A-G^{2}, \\
& \mathfrak{C}=A B-H^{2} \text {. } \\
& \sqrt{\mathfrak{f}}=G H-A F, \\
& \widehat{G}=H F-B G \text {, } \\
& \text { 埴 }=F G-C H \text {. } \\
& \mathbb{Q}=\mathfrak{A}, \mathfrak{b}, \mathfrak{C}, \sqrt{\mathfrak{f}}, \mathfrak{G}, \mathfrak{I} \\
& \overline{\omega \omega^{\prime}}=\eta \zeta^{\prime}-\eta^{\prime} \zeta, \quad \zeta \xi^{\prime}-\zeta^{\prime} \xi, \quad \xi \eta^{\prime}-\xi^{\prime} \eta
\end{aligned}
$$

we have

$W\left(\omega_{1}, \omega_{2}, Q\right) W\left(\omega_{3}, \omega_{4}, Q\right)-W\left(\omega_{1}, \omega_{3}, Q\right) W\left(\omega_{2}, \omega_{4}, Q\right)=W\left(\overline{\omega_{1} \omega_{4}}, \overline{\omega_{2} \omega_{3}}, \mathbb{Q}\right)$

of which we may notice also the particular eases

$$
\begin{array}{r}
W\left(\omega_{1}, \omega_{2}, Q\right) W\left(\omega_{3}, \omega_{3}, Q\right)-W\left(\omega_{1}, \omega_{3}, Q\right) W\left(\omega_{2}, \omega_{3}, Q\right)=W\left(\overline{\omega_{1} \omega_{3}}, \overline{\omega_{2} \omega_{3}}, \text { QR }\right) \ldots \\
W\left(\omega_{1}, \omega_{1}, Q\right) W\left(\omega_{2}, \omega_{2}, Q\right)-\left\{W\left(\omega_{1}, \omega_{2}, Q\right)\right\}^{2}=W\left(\underline{\overline{\omega_{1} \omega_{2}}}, \overline{\omega_{1} \omega_{2}}, \text { QR }\right) \ldots
\end{array}
$$

To these we may join the following formulæ, for the transformation of the function $W$. Suppose

$$
\begin{array}{lll}
\omega_{1}=a x_{1}+a^{\prime} y_{1}+a^{\prime \prime} z_{1}, & b x_{1}+b^{\prime} y_{1}+b^{\prime \prime} z_{1}, & c x_{1}+c^{\prime} y_{1}+c^{\prime \prime} z_{1} \\
\omega_{2}=a x_{2}+a^{\prime} y_{2}+a^{\prime \prime} z_{2}, & b x_{2}+b^{\prime} y_{2}+b^{\prime \prime} z_{2}, & c x_{2}+c^{\prime} y_{2}+c^{\prime \prime} z_{2},
\end{array}
$$

then, writing

$$
\begin{gathered}
g=a, \quad b, \quad c \\
g^{\prime}=a^{\prime}, \quad b^{\prime}, \quad c^{\prime}, \\
g^{\prime \prime}=a^{\prime \prime}, \quad b^{\prime \prime}, \quad c^{\prime \prime}, \\
p_{1}=x_{1}, y_{1}, z_{1} \\
p_{2}=x_{2}, y_{2}, z_{2},
\end{gathered}
$$

$\Theta=W(g, g, Q), W\left(g^{\prime}, g^{\prime}, Q\right), W\left(g^{\prime \prime}, g^{\prime \prime}, Q\right), W\left(g^{\prime}, g^{\prime \prime}, Q\right), W\left(g^{\prime \prime}, g, Q\right), W\left(g, g^{\prime}, Q\right)$ we have

$$
W\left(\omega_{1}, \omega_{2}, Q\right)=W\left(p_{1}, p_{2}, \Theta\right)
$$

Similarly, writing

$\Psi=W\left(\underline{\bar{g}^{\prime} g^{\prime \prime}}, \underline{\bar{g}^{\prime} g^{\prime \prime}}, \mathbb{Q}\right), \quad W\left(\underline{\overline{g^{\prime \prime} g}}, \underline{\underline{g^{\prime \prime} g}}, \mathbb{Q}\right), \quad W\left(\underline{\overline{g g^{\prime}}}, \overline{\overline{g g^{\prime}}}, \mathbb{Q}\right)$

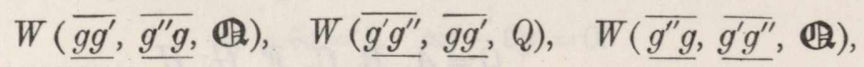

we have

$$
W\left(\overline{\omega_{1} \omega_{4}}, \overline{\omega_{2} \omega_{3}}, \mathbb{Q}\right)=W\left(\overline{p_{1} p_{4}}, \overline{p_{2} p_{3}}, \Psi\right)
$$

in which equations 1 may obviously be changed into $Q$. 
Section 2. Geometrical Applications.

Consider any three axes $A x, A y, A z$, and let $\lambda, \mu, \nu$ be the cosines of the inclinations of these lines to each other.

Let $\Lambda, \mathbf{M}, \mathbf{N}$ be the inclinations of the coordinate planes to each other; $l, m, n$ the inclination of the axes to the coordinate planes. Suppose, besides,

$$
\begin{aligned}
& \mathfrak{a}=1-\lambda^{2} \ldots \ldots \\
& \mathfrak{b}=1-\mu^{2}, \\
& \mathfrak{c}=1-\nu^{2}, \\
& \mathfrak{f}=\mu \nu-\lambda, \\
& \mathfrak{g}=\nu \lambda-\mu, \\
& \mathfrak{b}=\lambda \mu-\nu, \\
& k=1-\lambda^{2}-\mu^{2}-\nu^{2}+2 \lambda \mu \nu
\end{aligned}
$$

we have the following systems of equations:

$$
\begin{aligned}
& \sqrt{ }(\mathbf{b c}) \cos \Lambda=-\mathbf{f}, \quad \sqrt{ }(\mathbf{b c}) \sin \Lambda=\sqrt{ }(k), \quad \sqrt{ }(\mathbf{a}) \sin l=\sqrt{ }(k) . \\
& \sqrt{ }(\mathbf{c} \mathfrak{a}) \cos \mathbf{M}=-\mathfrak{g}, \quad \sqrt{ }(\mathfrak{c} \mathfrak{a}) \sin \mathbf{M}=\sqrt{ }(k), \quad \sqrt{ }(\mathfrak{b}) \sin m=\sqrt{ }(k) \\
& \sqrt{ }(\mathfrak{a b}) \cos \mathbf{N}=-\mathfrak{b}, \quad \sqrt{ }(\mathfrak{a b}) \sin \mathbf{N}=\sqrt{ }(k), \quad \sqrt{ }(\mathfrak{c}) \sin n=\sqrt{ }(k) \text {. } \\
& \mathfrak{a}+\nu \mathfrak{y}+\mu \mathfrak{g}=k, \\
& \nu \mathfrak{a}+\mathfrak{b}+\lambda \mathfrak{g}=0, \\
& \mu \mathfrak{a}+\lambda \mathfrak{b}+\mathfrak{g}=0 \text {. } \\
& \mathfrak{b}+\nu \mathfrak{b}+\mu \mathfrak{f}=0 \text {, } \\
& \nu \mathfrak{b}+\mathfrak{b}+\lambda \mathfrak{f}=k, \\
& \mu \mathfrak{b}+\lambda \mathfrak{b}+\mathbf{f}=0 \text {. } \\
& \mathfrak{g}+\nu \mathrm{f}+\mu \mathfrak{R}=0, \\
& \nu \mathfrak{g}+\mathfrak{f}+\lambda \mathfrak{r}=0, \\
& \mu \underline{a}+\lambda \mathfrak{f}+\mathfrak{c}=k \text {. } \\
& \mathrm{bc}-\mathrm{f}^{2}=k a \\
& \mathfrak{c a}-\mathfrak{a}^{2}=k b, \\
& \mathfrak{a b}-\mathfrak{b}^{2}=k c, \\
& \text { ab) }-a f=k f \text {, } \\
& \mathfrak{b f}-\mathfrak{b g}=k g \text {, } \\
& \mathfrak{f g}-\mathfrak{c b}=k h, \\
& a b c-a f^{2}-b g^{2}-c b^{2}+2 \mathfrak{f} \mathfrak{b}=k^{2}
\end{aligned}
$$


Imagine now a line $A O$, and let $\alpha, \beta, \gamma$ be the cosines of its inclinations to the three axes. Suppose also, $\theta, \phi, \chi$ being its inclinations to the coordinate planes, we write

$$
a=\frac{\sin \theta}{\sqrt{(\mathfrak{a})}}, \quad b=\frac{\sin \phi}{\sqrt{(\mathfrak{b})}}, \quad c=\frac{\sin \chi}{\sqrt{(\mathfrak{c})}}
$$

If we consider a point $P$ on the line $A O$, at a distance unity from the origin, we see immediately, by considering the projections in the directions perpendicular to the coordinate planes, that the coordinates of this point are $a, b, c$. By projecting on the three axes and on the line $A O$, we then obtain the equations

$$
\begin{aligned}
& \alpha=a+\nu b+\mu c \\
& \beta=\nu a+b+\lambda c, \\
& \gamma=\mu a+\lambda b+c, \\
& 1=\alpha a+\beta b+\gamma c
\end{aligned}
$$

from which we obtain

$$
\begin{aligned}
& k a=\mathfrak{a} \alpha+\mathfrak{b} \beta+\mathfrak{g} \gamma \\
& k b=\mathfrak{b} \alpha+\mathfrak{b} \beta+\mathfrak{f} \gamma, \\
& k c=\mathfrak{g} \alpha+\mathfrak{f} \beta+\mathfrak{c} \gamma, \\
& 1=\alpha a+\beta b+\gamma c
\end{aligned}
$$

and hence

$$
\begin{aligned}
& 1=a^{2}+b^{2}+c^{2}+2 \lambda b c+2 \mu a c+2 \nu a b \ldots \ldots \\
& k=\mathfrak{a} \alpha^{2}+\mathbf{b} \beta^{2}+\mathfrak{c} \gamma^{2}+2 \mathfrak{f} \beta \gamma+2 \mathfrak{g} \alpha \boldsymbol{\gamma}+2 \mathfrak{b} \alpha \beta
\end{aligned}
$$

Hence writing

$$
\begin{aligned}
& a, b, c=t \\
& \alpha, \beta, \gamma=\tau
\end{aligned}
$$

$1,1,1, \lambda, \mu, \nu=q$

$$
\mathfrak{a}, \mathfrak{b}, \mathfrak{c}, \mathfrak{f}, \mathfrak{a}, \mathfrak{b}=\mathfrak{q}
$$

we have the equations

$$
\begin{aligned}
& 1=W(t, t, \mathfrak{q}) \\
& k=W(\tau, \tau, \mathfrak{q})
\end{aligned}
$$

Let $A O^{\prime}$ be any other line, and $\delta$ its inclination to $A O: \alpha^{\prime}, \beta^{\prime}, \gamma^{\prime}, a^{\prime}, b^{\prime}, c^{\prime}$, the quantities corresponding to $\alpha, \beta, \gamma, a, b, c$, and similarly $t^{\prime}, \tau^{\prime}$ to $t, \tau$. We have of course

$$
\begin{aligned}
& 1=W\left(t^{\prime}, t^{\prime}, q\right) \\
& k=W\left(\tau^{\prime}, \tau^{\prime}, \mathfrak{q}\right)
\end{aligned}
$$


We have besides, by projecting on the line $A O^{\prime}$, the equation

$$
\cos \delta=\alpha^{\prime} a+\beta^{\prime} b+\gamma^{\prime} c
$$

or the analogous one

$$
\cos \delta=\alpha^{\prime} a+\beta^{\prime} b+\gamma^{\prime} c
$$

From either of which we deduce

$$
\begin{array}{r}
\cos \delta=a a^{\prime}+b b^{\prime}+c c^{\prime}+\lambda\left(b c^{\prime}+b^{\prime} c\right)+\mu\left(c a^{\prime}+c^{\prime} a\right)+\nu\left(a b^{\prime}+a^{\prime} b\right) \\
k \cos \delta=\mathfrak{a} \alpha \alpha^{\prime}+\mathfrak{b} \beta \beta^{\prime}+\mathfrak{c} \gamma \gamma^{\prime}+\mathfrak{f}\left(\beta \gamma^{\prime}+\beta^{\prime} \gamma\right)+\mathfrak{g}\left(\gamma^{\alpha^{\prime}}+\gamma^{\prime} \alpha^{\prime}\right)+\mathfrak{b}\left(\alpha \beta+\alpha^{\prime} \beta\right)
\end{array}
$$

which may otherwise be written

$$
\begin{aligned}
\cos \delta & =W\left(t, t^{\prime}, q\right) \\
k \cos \delta & =W\left(\tau, \tau^{\prime}, \mathfrak{q}\right)
\end{aligned}
$$

or again, observing the equations which connect the quantities $t, \tau$,

$$
\begin{aligned}
& \cos \delta=\frac{W\left(t, t^{\prime}, q\right)}{\left.\sqrt{\left\{W(t, t, q) W\left(t^{\prime}, t^{\prime}, q\right)\right.}\right\}} \\
& \cos \delta=\frac{W\left(\tau, \boldsymbol{\tau}^{\prime}, \mathfrak{q}\right)}{\sqrt{ }\left\{W(\tau, \tau, \mathfrak{q}) W\left(\boldsymbol{\tau}^{\prime}, \boldsymbol{\tau}^{\prime}, \mathfrak{q}\right)\right\}}
\end{aligned}
$$

forms which, though more complicated, have certain advantages; for instance, we derive immediately from them the new equations

$$
\begin{aligned}
& \sin \delta=\frac{\sqrt{ }\left\{W\left(\overline{t_{t^{\prime}}}, \overline{t_{t^{\prime}}, q}\right)\right\}}{\sqrt{ }\left\{W(t, t, \mathrm{q}) W\left(t^{\prime}, t^{\prime}, \mathrm{q}\right)\right\}} \\
& \sin \delta=\frac{\sqrt{ }\left\{k W\left(\overline{\tau \tau^{\prime}}, \overline{\tau^{\prime}}, q\right)\right\}}{\sqrt{ }\left\{W(\tau, \tau, \mathfrak{q}) W\left(\tau^{\prime}, \tau^{\prime}, \mathfrak{q}\right)\right\}}
\end{aligned}
$$

written more simply thus

$$
\begin{aligned}
\sin \delta & =W\left(\underline{\overline{t t^{\prime}}}, \overline{\overline{t t^{\prime}}}, \mathfrak{q}\right) \ldots \\
\sqrt{ } k \sin \delta & =\sqrt{ }\left\{W\left(\overline{\overline{\tau \tau^{\prime}}}, \overline{\overline{\tau \tau^{\prime}}}, \mathrm{q}\right)\right\}
\end{aligned}
$$

to these we may join

$$
\begin{aligned}
\cot \delta & =\frac{W\left(t, t^{\prime}, \mathrm{q}\right)}{\left.\sqrt{\left\{W\left(\overline{t t^{\prime}}, \overline{t t^{\prime}}, q\right)\right.}\right)} \cdots \\
\sqrt{ } k \cot \delta & =\frac{W\left(\tau, \tau^{\prime}, q\right)}{\sqrt{\left\{W\left(\overline{\tau \tau^{\prime}}, \overline{\tau \tau^{\prime}}, q\right)\right\}}}
\end{aligned}
$$

Section 3. On Spherical Coordinates.

Consider the points $X, Y, Z$, on the surface of a sphere, as the intersections of the three axes of the preceding section, with a sphere having its centre in the origin. It is evident that $\lambda, \mu, \nu$ are the cosines of the sides of the spherical triangle $X Y Z$, c. 
$\Lambda, \mathrm{M}, \mathrm{N}$ are its sides, $l, m, n$ are the perpendiculars from the angles upon the opposite sides. Let $P$ be the point where the line $A O$ intersects the sphere: the position of the point $P$ may be determined by means of the ratios $\xi: \eta: \zeta$, supposing $\xi, \eta, \zeta$ denote quantities proportional to the $\alpha, \beta, \gamma$ of the preceding section, i.e.

$$
\xi: \eta: \zeta=\cos P X: \cos P Y: \cos P Z
$$

or again, by means of the ratios $x: y: z$, supposing $x, y, z$ denote quantities proportional to the $a, b, c$ of the preceding section, i.e.

$$
x: y: z=\frac{\sin P x}{\sin X}: \frac{\sin P y}{\sin Y}: \frac{\sin P z}{\sin Z}
$$

$(P x, P y, P z$ are the perpendiculars from $P$ on the sides of the spherical triangle $X Y Z)$.

These last equations may be otherwise written,

$$
\begin{aligned}
& \frac{x \sin X}{y \sin Y}=\frac{\sin P Z Y}{\sin P Z X} \\
& \frac{y \sin Y}{z \sin Z}=\frac{\sin P X Z}{\sin P X Y}, \\
& \frac{z \sin Z}{x \sin X}=\frac{\sin P Y X}{\sin P Y Z} .
\end{aligned}
$$

The ratios $\xi: \eta: \zeta$, or $x: y: z$, are termed the spherical coordinate ratios of the point $P$. The two together may be termed conjoint systems: the first may be termed the cosine system, and the second the sine system. The coordinates of the two systems are evidently connected by

or

$$
\begin{aligned}
& \xi: \eta: \zeta=x+\nu y+\mu z: \nu x+y+\lambda z: \mu x+\lambda y+z \\
& x: y: z=\mathbf{a} \xi+\mathfrak{b} \eta+\mathfrak{a} \zeta: \mathfrak{b} \xi+\mathfrak{b} \eta+\mathfrak{f} \zeta: \mathfrak{a} \xi+\mathfrak{f} \eta+\mathfrak{c} \zeta .
\end{aligned}
$$

The systems may conveniently be represented by the single letters

$$
\begin{aligned}
& \omega=\xi, \eta, \zeta \\
& p=x, y, z
\end{aligned}
$$

Fundamental formula of spherical coordinates; distance of two points.

Let $P, P^{\prime}$ be the points, $\delta$ their distance, $\omega, p$ the conjoint coordinate systems of the first point, $\omega^{\prime}, p^{\prime}$ of the second; we have obviously

$$
\begin{aligned}
& \cot \delta=\frac{W\left(p, p^{\prime}, q\right)}{\sqrt{ }\left\{W(p, p, q) W\left(p^{\prime}, p^{\prime}, q\right)\right\}} \\
& \sin \delta=\frac{\sqrt{ }\left\{W\left(\overline{p p^{\prime}}, \overline{p p^{\prime}}, q\right)\right\}}{\sqrt{ }\left\{W(p, p, q) W\left(p^{\prime}, p^{\prime}, q\right)\right\}}, \\
& \cot \delta=\frac{W\left(p, p^{\prime}, q\right)}{\sqrt{ }\left\{W\left(\underline{p p^{\prime}}, \overline{p p^{\prime}} q\right)\right\}} \text {; }
\end{aligned}
$$




$$
\begin{aligned}
\cos \delta & =\frac{W\left(\omega, \omega^{\prime}, \mathfrak{q}\right)}{\sqrt{ }\left\{W(\omega, \omega, \mathfrak{q}) W\left(\omega^{\prime}, \omega^{\prime}, \mathfrak{q}\right)\right\}} \\
\frac{1}{\sqrt{ } k} \sin \delta & =\frac{\sqrt{ }\left\{W\left(\overline{\omega \omega^{\prime}}, \overline{\omega \omega^{\prime}}, q\right)\right\}}{\sqrt{ }\left\{W(\omega, \omega, \mathfrak{q}) W\left(\omega^{\prime}, \omega^{\prime}, \mathfrak{q}\right)\right\}}, \\
\sqrt{ } k\{\cot \delta & =\frac{W\left(\omega, \omega^{\prime}, \mathfrak{q}\right)}{\sqrt{ }\left\{W\left(\underline{\omega \omega^{\prime}}, \underline{\omega \omega^{\prime}}, \mathfrak{q}\right)\right\}} .
\end{aligned}
$$

Equation of a great Circle.

Let the conjoint coordinate systems of the pole be

$$
\begin{aligned}
& e=a, b, c \\
& \epsilon=\alpha, \beta, \gamma
\end{aligned}
$$

then, expressing that the distance of any point $P$ in the locus from the pole is equal to $90^{\circ}$, we have immediately the equations

$$
\begin{aligned}
& W(p, e, q)=0 \\
& W(\omega, \epsilon, \mathfrak{q})=0
\end{aligned}
$$

which may otherwise be written in the forms

$$
\begin{aligned}
& a \xi+b \eta+c \zeta=0 \\
& a x+\beta y+\gamma \zeta=0
\end{aligned}
$$

or the equation of a great circle is linear in either coordinate system. Conversely, any linear equation belongs to a great circle.

Suppose the equation given in the form

$$
A \xi+B \eta+C \zeta=0
$$

or by an equation between cosine coordinate ratios:-the sine system for the pole is given by

$$
e=A, B, C
$$

and the cosine system by

$$
\epsilon=A+\nu B+\mu C, \quad \nu A+B+\lambda C, \quad \mu A+\lambda B+C .
$$

Suppose the circle given by an equation between sine coordinates, or in the form

$$
\mathrm{A} x+\mathrm{B} y+\mathrm{C} z=0
$$

the cosine system of coordinates for the pole is given by

$$
\epsilon=\mathrm{A}, \mathrm{B}, \mathrm{C}
$$

and the sine system by

$$
e=\mathfrak{a A}+\mathfrak{b B}+\mathfrak{a C}, \quad \mathfrak{b A}+\mathfrak{b B}+\mathfrak{f C}, \quad \mathfrak{g A}+\mathfrak{f B}+\mathfrak{c C}
$$


It is hardly necessary to observe, that if

$$
\begin{aligned}
& A \xi+B \eta+C \zeta=0 \\
& \mathrm{~A} x+\mathrm{B} y+\mathrm{C} z=0
\end{aligned}
$$

represent the same great circle,

$$
\begin{aligned}
& \mathrm{A}: \mathrm{B}: \mathrm{C}=A+\nu B+\mu C: \nu A+B+\lambda C: \mu A+\lambda B+C \\
& A: B: C=\mathfrak{a A}+\mathfrak{b B}+\mathfrak{g C}: \mathfrak{b} \mathrm{A}+\mathfrak{b B}+\mathfrak{f C}: \mathfrak{g} \mathrm{A}+\mathfrak{f B}+\mathfrak{r C}
\end{aligned}
$$

Inclination of two great Circles.

Let the equations of these be

$$
\begin{aligned}
& \left\{\begin{aligned}
A \xi+B \eta+C \zeta & =0 \ldots \ldots \ldots \ldots \ldots \ldots \ldots \ldots \ldots \ldots \ldots \ldots \ldots \ldots \ldots \ldots \ldots \ldots \ldots \ldots \ldots \ldots \ldots \ldots \ldots
\end{aligned}\right. \\
& \left\{\begin{array}{r}
A^{\prime} \xi+B^{\prime} \eta+C^{\prime} \zeta=0 \\
\text { or } A^{\prime} x+B^{\prime} y+C^{\prime} z=0
\end{array}\right.
\end{aligned}
$$

and let $e, \epsilon$, have the same values as above, and $e^{\prime}, \epsilon^{\prime}$, corresponding ones. To obtain the inclination of the two circles, we have only, in the formulæ given above for the distance of two points, to change $p, p^{\prime}, \omega, \omega^{\prime}$, into $e, e^{\prime}, \epsilon, \epsilon^{\prime}$.

The distance of a point from a given circle may be found with equal facility; for this is evidently the complement of the distance of the point from the pole of the circle. In like manner we may find the condition that two great circles intersect at right angles, \&c.

There are evidently a whole class of formulæ, not by any means peculiar to the present system of coordinates, such as

$$
A x+B y+C z-s\left(A^{\prime} x+B^{\prime} y+C^{\prime} z\right)
$$

for the equation of a great circle subjected to pass through the points of intersection of

$$
A x+B y+C z=0, \quad A^{\prime} x+B^{\prime} y+C^{\prime} z=0 .
$$

Again,

$$
\left|\begin{array}{lll}
x, & y, & z \\
a, & b, & c \\
a^{\prime}, & b^{\prime}, & c^{\prime}
\end{array}\right|=0
$$

for the equation of the great circle which passes through the points given by the sine systems $a: b: c$ and $a^{\prime}: b^{\prime}: c^{\prime}$, \&c., and which are obtained so easily that it is not worth while writing down any more of them.

\section{Transformation of Coordinates.}

Let $X_{1}, Y_{1}, Z_{1}$, be the new points of reference, and suppose $X_{1}$ is given by the conjoint systems $e=a, b, c, \epsilon=\alpha, \beta, \gamma$; and similarly $Y_{1}, Z_{1}$, by the analogous systems $e^{\prime}, \epsilon^{\prime} ; e^{\prime \prime}, \epsilon^{\prime \prime}$. 
Suppose, as before, $P$ is given by one of the systems $\omega, p$; and let $\omega_{1}, p_{1}$ be the new systems which determine the position of $P$ with reference to $X_{1}, Y_{1}, Z_{1}$.

In the first place, $\lambda_{1}, \mu_{1}, \nu_{1}$, are given by the formulæ

$$
\begin{aligned}
& \lambda_{1}=\frac{W\left(e^{\prime}, e^{\prime \prime}, q\right)}{\sqrt{\left\{W\left(e^{\prime}, e^{\prime}, q\right) W\left(e^{\prime \prime}, e^{\prime \prime}, q\right)\right\}}}=\frac{W\left(\epsilon^{\prime}, \epsilon^{\prime \prime}, \mathfrak{q}\right)}{\sqrt{\left\{W\left(\epsilon^{\prime}, \epsilon^{\prime}, \mathfrak{q}\right) W\left(\epsilon^{\prime \prime}, \epsilon^{\prime \prime}, \mathfrak{q}\right)\right\}}} \cdot \\
& \mu_{1}=\frac{W\left(e^{\prime \prime}, e, q\right)}{\sqrt{\left\{W\left(e^{\prime \prime}, e^{\prime \prime}, q\right) W(e, e, q)\right\}}}=\frac{W\left(\epsilon^{\prime \prime}, \epsilon, \mathfrak{q}\right)}{\sqrt{\left\{W\left(\epsilon^{\prime \prime}, \epsilon^{\prime \prime}, \mathfrak{q}\right) W(\epsilon, \epsilon, \mathfrak{q})\right\}},} \\
& \nu_{1}=\frac{W\left(e, e^{\prime}, \mathfrak{q}\right)}{\sqrt{\left\{W(e, e, q) W\left(e^{\prime}, e^{\prime}, q\right)\right\}}}=\frac{W\left(\epsilon, \epsilon^{\prime}, \mathfrak{q}\right)}{\sqrt{\left\{W(\epsilon, \epsilon, \mathfrak{q}) W\left(\epsilon^{\prime}, \epsilon^{\prime}, \mathfrak{q}\right)\right\}}} .
\end{aligned}
$$

The system $\omega_{1}$ is evidently given immediately by

$$
\begin{aligned}
\xi_{1}: \eta_{1}: \zeta_{1} & =\frac{W(e, p, q)}{\sqrt{ }\{W(e, e, q)\}}: \frac{W\left(e^{\prime}, p, q\right)}{\sqrt{ }\left\{W\left(e^{\prime}, e^{\prime}, q\right)\right\}}: \frac{W\left(e^{\prime \prime}, p, q\right)}{\sqrt{ }\left\{W\left(e^{\prime \prime}, e^{\prime \prime}, q\right)\right\}} \\
& =\frac{W(\epsilon, \omega, \mathfrak{q})}{\sqrt{ }\{W(\epsilon, \epsilon, q)\}}: \frac{W\left(\epsilon^{\prime}, \omega, \mathfrak{q}\right)}{\sqrt{ }\left\{W\left(\epsilon^{\prime}, \epsilon^{\prime}, \mathfrak{q}\right)\right\}}: \frac{W\left(\epsilon^{\prime \prime}, \omega, \mathfrak{q}\right)}{\sqrt{ }\left\{W\left(\epsilon^{\prime \prime}, \epsilon^{\prime \prime}, \mathfrak{q}\right)\right\}}
\end{aligned}
$$

and from these we may obtain the system $p_{1}$, by means of the formulæ

$$
x_{1}: y_{1}: z_{1}=\mathfrak{a}_{1} \xi_{1}+\mathfrak{b}_{1} \eta_{1}+\mathfrak{a}_{1} \xi_{1}: \mathfrak{b}_{1} \xi_{1}+\mathfrak{b}_{1} \eta_{1}+\mathfrak{f}_{1} \xi_{1}: \mathfrak{a}_{1} \xi_{1}+\mathfrak{f}_{1} \eta_{1}+\mathfrak{c}_{1} \zeta_{1}
$$

This requires some further development however. We must in the first place form the system $\mathfrak{a}_{1}, \mathfrak{b}_{1}, \mathfrak{r}_{1}, \mathfrak{f}_{1}, \mathfrak{a}_{1}, \mathfrak{b}_{1}$ : this is done immediately from the formulæ of Sect. 2 , and we have

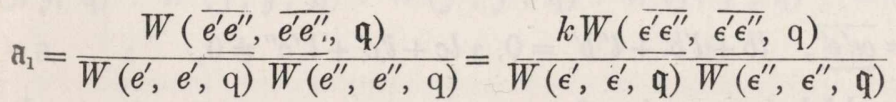

$$
\begin{aligned}
& \mathfrak{b}_{1}=\frac{W\left(\overline{e^{\prime \prime} e}, \overline{e^{\prime \prime} e}, \mathfrak{q}\right)}{W\left(e^{\prime \prime}, e^{\prime \prime}, q\right)} \overline{W\left(e^{\prime \prime}, e, q\right)}=\frac{k W\left(\overline{\epsilon^{\prime \prime} \epsilon}, \overline{\epsilon^{\prime \prime} \epsilon}, q\right)}{W\left(\epsilon^{\prime \prime}, \epsilon^{\prime \prime}, \bar{q}\right)} \overline{W(\epsilon, \epsilon, \mathfrak{q})}, \\
& \mathfrak{c}_{1}=\frac{W\left(\overline{e e^{\prime}}, \overline{e e^{\prime}}, \mathfrak{q}\right)}{W(e, e, \bar{q}) \bar{W}\left(e^{\prime}, e^{\prime}, \mathfrak{q}\right)}=\frac{k W\left(\overline{\epsilon \epsilon^{\prime}}, \overline{\epsilon \epsilon^{\prime}}, q\right)}{W(\epsilon, \epsilon, \mathfrak{q}) W\left(\epsilon^{\prime}, \epsilon^{\prime}, \mathfrak{q}\right)}, \\
& \mathrm{f}_{1}=\frac{W\left(\overline{e^{\prime \prime} e}, \overline{e e^{\prime}}, \mathfrak{q}\right)}{W(e, e, q) \sqrt{ }\left(W\left(e^{\prime}, e^{\prime}, q\right) W\left(e^{\prime \prime}, e^{\prime \prime}, q\right)\right\}}=\frac{k W\left(\overline{\epsilon^{\prime \prime} \epsilon}, \overline{\epsilon \epsilon^{\prime}}, q\right)}{W(\epsilon, \epsilon, \mathfrak{q}) \sqrt{ }\left\{W\left(\epsilon^{\prime}, \epsilon^{\prime}, \mathfrak{q}\right) W\left(\epsilon^{\prime \prime}, \epsilon^{\prime \prime}, \mathfrak{q}\right)\right\}},
\end{aligned}
$$

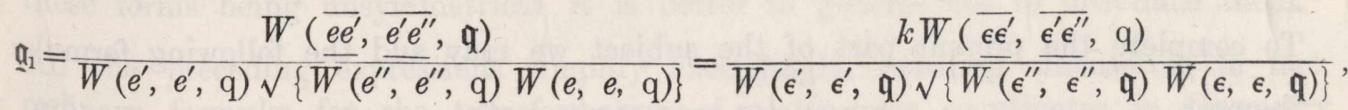

$$
\begin{aligned}
& \mathfrak{b}_{1}=\frac{W\left(\overline{e^{\prime} e^{\prime \prime}}, \overline{e^{\prime \prime} e}, \mathfrak{q}\right)}{W\left(e^{\prime \prime}, e^{\prime \prime}, q\right) \sqrt{ }\left\{\bar{W}(e, e, q) W\left(e^{\prime}, e^{\prime}, \mathfrak{q}\right)\right\}}=\frac{k W\left(\overline{\epsilon^{\prime} \epsilon^{\prime \prime}}, \overline{\epsilon^{\prime \prime} \epsilon}, q\right)}{W\left(\epsilon^{\prime \prime}, \epsilon^{\prime \prime}, \mathfrak{q}\right) \sqrt{\left\{\bar{W}(\epsilon, \bar{\epsilon}, \mathfrak{q}) W\left(\epsilon^{\prime}, \epsilon^{\prime}, \mathfrak{q}\right)\right.} .} .
\end{aligned}
$$




$$
\begin{aligned}
& x_{1}: y_{1}: z_{1}=\sqrt{ }\{W(e, \mathrm{~B}, \mathrm{q})\} \times \\
& \left\{W(e, p, q) W\left(\overline{e^{\prime} e^{\prime \prime}}, \overline{e^{\prime} e^{\prime \prime}}, q\right)+W\left(e^{\prime}, p, q\right) W\left(\underline{e^{\prime} e^{\prime \prime}}, \overline{e^{\prime \prime} e}, \mathfrak{q}\right)+W\left(\left(e^{\prime \prime}, p, \mathfrak{q}\right) W\left(\overline{e^{\prime} e^{\prime \prime}}, \overline{e e^{\prime}}, \mathfrak{q}\right)\right\}\right. \\
& : \sqrt{ }\left\{W\left(e^{\prime}, e^{\prime}, q\right)\right\} \times \\
& \left\{W(e, p, q) W\left(\underline{e^{\prime \prime} \cdot e}, \overline{e^{\prime} e^{\prime \prime}}, q\right)+W\left(e^{\prime}, p, q\right) W\left(\overline{e^{\prime \prime} e}, \overline{e^{\prime \prime} e}, q\right)+W\left(\underline{e^{\prime \prime}}, p, q\right) W\left(\overline{e^{\prime \prime} e,}, \overline{e e^{\prime}}, q\right)\right\} \\
& : \sqrt{ }\left\{W\left(e^{\prime \prime}, e^{\prime \prime}, q\right)\right\} \times \\
& \left\{W(e, p, q) W\left(\overline{e e^{\prime}}, \overline{e^{\prime} e^{\prime \prime}}, q\right)+W\left(e^{\prime}, p, q\right) W\left(\overline{e e^{\prime}}, \overline{e^{\prime \prime} e}, \mathfrak{q}\right)+W\left(e^{\prime \prime}, p, q\right) W\left(\overline{e e^{\prime}}, \overline{e e^{\prime}}, q \mathfrak{q}\right)\right\} ; \\
& \text { these may be reduced to the very simple form } \\
& \begin{aligned}
x_{1}: y_{1}: z_{1}= & \sqrt{ }\{W(e, e, q)\} W\left(\overline{\left(e^{\prime} e^{\prime \prime}\right.}, \omega, q\right) \\
: & \sqrt{ }\left\{W\left(e^{\prime}, e^{\prime}, q\right)\right\} W\left(\overline{e^{\prime \prime} e}, \omega, q\right), \\
: & \sqrt{ }\left\{W\left(e^{\prime \prime}, e^{\prime \prime}, q\right)\right\} W\left(\overline{e^{\prime}}, \omega, q\right)
\end{aligned}
\end{aligned}
$$

and in like manner we obtain

$$
\begin{aligned}
x_{1}: y_{1}: z_{1}= & \sqrt{ }\{W(\boldsymbol{\epsilon}, \boldsymbol{\epsilon}, \mathfrak{q})\} W\left(\overline{\overline{\epsilon^{\prime} \epsilon^{\prime}}}, p, q\right) \\
: & \sqrt{ }\left\{W\left(\boldsymbol{\epsilon}^{\prime}, \epsilon^{\prime}, \mathfrak{q}\right)\right\} W\left(\overline{\epsilon^{\prime \prime} \epsilon}, p, q\right) \\
: & \sqrt{ }\left\{W\left(\epsilon^{\prime \prime}, \boldsymbol{\epsilon}^{\prime \prime}, \mathfrak{q}\right)\right\} W\left(\overline{\overline{\epsilon \epsilon^{\prime}}}, p, q\right) .
\end{aligned}
$$

It will be as well to indicate the steps of this reduction. Consider the quantity in \{\} in the first line of the equation which gives the ratios $x_{1}: y_{1}: z_{1}$; and suppose for a moment $\overline{e^{\prime} e^{\prime \prime}}=l, m, n$, \&c.: then, selecting the portion of the expression which is multiplied by $a$, this is

$$
=a l\left\{l(a \xi+b \eta+c \zeta)+l^{\prime}\left(a^{\prime} \xi+b^{\prime} \eta+c^{\prime} \zeta\right)+l^{\prime \prime}\left(a^{\prime \prime} \xi+b^{\prime \prime} \eta+c^{\prime \prime} \zeta\right)\right\},
$$

or, since

$$
l a+l^{\prime} a^{\prime}+l^{\prime \prime} a^{\prime \prime}=\overline{e e^{\prime} e^{\prime \prime}}, \quad l b+l^{\prime} b^{\prime}+l^{\prime \prime} b^{\prime \prime}=0, \quad l c+l^{\prime} c^{\prime}+l^{\prime \prime} c^{\prime \prime}=0,
$$

this reduces itself to $\overline{e e^{\prime} e^{\prime \prime}} \cdot a l \xi$, which is a term of

$$
\overline{e e^{\prime} e^{\prime \prime}} W\left(\overline{e^{\prime} e^{\prime \prime}}, \omega, \mathfrak{q}\right) \text {; }
$$

and by comparing the remaining terms in the same manner, it would be seen that the whole reduces itself to

$$
\overline{e e^{\prime} e^{\prime \prime}} W\left(\underline{e^{\prime} e^{\prime \prime}}, \omega, \mathbf{q}\right)
$$

whence the formulæ in question.

The formulæ (86), (87), (91), (92), completely resolve the problem of the transformation of coordinates; they determine respectively $p_{1}$ from $p$ or $\omega$, $\omega_{1}$ from $p$ or $\omega$.

To complete the present part of the subject we may add the following formulæ.

Suppose

$$
\begin{aligned}
x_{1}: y_{1}: z_{1} & =a x_{1}+\mathrm{a}^{\prime} y_{1}+\mathrm{a}^{\prime \prime} z_{1} \\
& : \mathrm{b} x_{1}+\mathrm{b}^{\prime} y_{1}+\mathrm{b}^{\prime \prime} y_{1}, \\
& : \mathrm{c} x_{1}+\mathrm{c}^{\prime} y_{1}+\mathrm{c}^{\prime \prime} z_{1},
\end{aligned}
$$


which we see from the preceding formulæ is the form of the relation between the systems $p_{1}$ and $p$. And suppose, as before, $\lambda_{1}, \mu_{1}, \nu_{1}$ are the eosines of the distances of the new points of reference $\mathrm{X}_{1}, \mathrm{Y}_{1}, \mathrm{Z}_{1}$.

We can immediately determine the relations that must exist between these coefficients, in order that they may actually denote such a transformation. For this purpose write

$$
\begin{aligned}
& \mathrm{a}, \mathrm{b}, 、 \mathrm{c}=j \\
& a^{\prime}, \quad b^{\prime}, \quad c^{\prime}=j^{\prime}, \\
& a^{\prime \prime}, \quad b^{\prime \prime}, \quad c^{\prime \prime}=j^{\prime \prime} \text {. }
\end{aligned}
$$

Then the distance between the point $P$ and any other point $P^{\prime}$ is given by the formula

$$
\cos \delta=\frac{W\left(p, p^{\prime}, q\right)}{\cdot \sqrt{ }\left\{W(p, p, q) W\left(p^{\prime}, p^{\prime}, q\right)\right\}}=\frac{W\left(p_{1}, p_{1}^{\prime}, \Theta\right)}{\sqrt{ }\left\{W\left(p_{1}, p_{1}, \Theta\right) W\left(p_{1}, p_{1}^{\prime}, \Theta\right)\right\}} \cdots
$$

where

$\Theta=W(j, j, q), \quad W\left(j^{\prime}, j^{\prime}, q\right), \quad W\left(j^{\prime \prime}, j^{\prime \prime}, q\right), \quad W\left(j^{\prime}, j^{\prime \prime}, q\right), \quad W\left(j^{\prime \prime}, j, q\right), \quad W\left(j, j^{\prime}, q\right) \ldots(96)$.

But we must evidently have

$$
\cos \delta=\frac{W\left(p_{1}, p_{1}^{\prime}, q_{1}\right)}{\sqrt{ }\left\{W\left(p_{1}, p_{1}, q_{1}\right) W\left(p_{1}^{\prime}, p_{1}^{\prime}, q_{1}\right)\right\}}
$$

or the quantities $\Theta$ must be proportional to the quantities q, i.e.

$$
\begin{aligned}
& W(j, j, \mathrm{q}): W\left(j^{\prime}, j^{\prime}, \mathrm{q}\right): W\left(j^{\prime \prime}, j^{\prime \prime}, \mathrm{q}\right): W\left(j^{\prime}, j^{\prime \prime}, \mathrm{q}\right): W\left(j^{\prime \prime}, j, \mathrm{q}\right): W\left(j^{\prime \prime}, j, \mathrm{q}\right)
\end{aligned}
$$

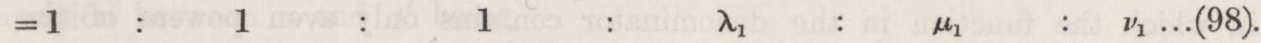

And in precisely the same manner, if instead of $x, y, z, x_{1}, y_{1}, z_{1}$, in the above formulæ, we had had $\xi, \eta, \zeta: \xi_{1}, \eta_{1}, \zeta_{1}$, the result would have been

$$
\begin{aligned}
& W(j, j, \mathfrak{q}): W\left(j^{\prime}, j^{\prime}, \mathfrak{q}\right): W\left(j^{\prime \prime}, j^{\prime \prime}, \mathfrak{q}\right): W\left(j^{\prime}, j^{\prime \prime}, \mathfrak{q}\right): W\left(j^{\prime \prime}, j, \mathfrak{q}\right): W\left(j^{\prime \prime}, j, \mathfrak{q}\right)
\end{aligned}
$$

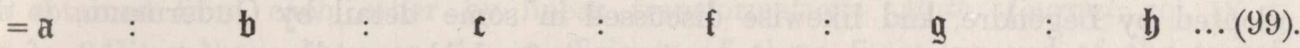

It is hardly necessary to remark, that throughout the preceding formulæ an expression, such as $W\left(p, p^{\prime}, q\right)$, is propartional to either of the quantities

$$
x \xi^{\prime}+y \eta^{\prime}+z \zeta^{\prime} \text { or } x^{\prime} \xi+y^{\prime} \eta+z^{\prime} \zeta,
$$

and may be changed into one of these multiplied by an arbitrary constant, which may be always made to disappear by a corresponding change in another quantity of the same form: thus, for instance,

$$
\frac{W\left(p, p^{\prime}, q\right)}{W(p, p, q)}=\frac{x^{\prime} \xi+y^{\prime} \eta+z^{\prime} \zeta}{x \xi+y \eta+z \zeta}
$$

but these forms being unsymmetrical, it is better in general not to introduce them.

All the preceding expressions simplify exceedingly, reducing themselves in fact to the ordinary formulæ for the transformation of rectangular coordinates in Geometry of three dimensions, for the case where the triangle $X Y Z$ has its sides and angles right angles. As this presents no difficulty, I shall not enter upon it at present. 